see that the application was not efficient; and Legouest's ease was one which probably could not have been cured by compression under any circumstances; nor was the compression very perfectly and methodically carried out. Now, gluteal aneurism presents almost every feature which is enzouraging for compression. The tumour, if the sac has not burst, is usually of no great size, it does not encroach on the abdomen, and any part of the aorta or common iliac is accessible to pressure. Surely the first step in the treatment of such a case should te to try the effect of methodical compression of the aorta or common iliac artery. If this can be done sucessfully without chloroform, which in a case not very seute might, I think, be possible, the patient would not be exposed to any serions danger; and we have too many histories now of aneurisms, even of large size, being cured by intermittent pressure, to deny the possibility; while, on the other band, the history of such cases as I have referred to, in Dubrueil's and Syme's patients, proves that it is not erery case of gluteal aneurism which justifies dangerous measures. If compression without chloroform is not possible, then the cure of the aneurism by total compression under chloroform should be attempted, and will in all probability ibe obtained in a large proportion of cases. If it fail, it may We repeated, in combination with coagulating injection, or galrano-puncture may be, and should be, tried. It is only After the failure of all these measures that the question of a capital operation occurs, and that question is no doubt a yery serious one. I confess that I should be disposed to sestrict the operation on the internal iliac artery to those eases in which there seem to be grave doubts whether the mouth of the aneurism is not inside the pelvis; whenever it so plainly outside that cavity I would perform Anel's operation, and if on trial this should prove impossible, I would apen the sac. It must be recollected that the efficient p*essure which the abdominal tourniquet now enables us to apply to the common iliac artery would rob the old operation of much of its danger.

There are only two cases of arterio-venous aneurism in 3he buttock on record, and in both the sciatic was the artery ateded. At least this seems more probable, but there is some discrepancy between various accounts of Nélaton's ease as to whether it was of the gluteal or the sciatic vessels. $\mathbb{N}$ élaton's patient was cured by the injection of perchloride 2 iron. Riberi's derived benefit from compression, but \$as not cured. The example of this case of Nélaton's woald encourage a surgeon to attempt the cure of this disby injection of perchloride of iron, or, perhaps, by galpano-puncture with the abdominal tourniquet; but on the failure of such attempts, the old operation would be the aly trustworthy method, if the symptoms were sufficiently sargent to justify the risk.

The conclusions, then, to which present experience seems to $m e$ to point in the treatment of gluteal aneurism are as Jollows :-

I. Gluteal aneurisms, both traumatic and spontaneous, sire very favourably circumstanced for the treatment by either rapid or gradual compression, applied to the aorta or caremon iliac.

2. If this treatment does not succeed by itself, it may be sapplemented by coagulating injection or galvano-puncture, serformed while the patient is narcotised and the circulajion commanded.

3. When such treatment fails, and particularly in aneusisms with imperfect or ruptured sacs where it is not inincated, the internal iliac must be tied when the surgeon thinks that he cannot find the artery outside the pelvis. But when the artery is accessible, the old operation, or the vperation of Anel, should be practised, according to the size and extent of the tumour.

4. The ligature of the internal iliac artery is liable bo failure in cases of spontaneous aneurism from a diseased condition of the coats of the artery, and should al ways be avoided when other means of treatment are availsble.

Plevro-pneumonia has, we understand, made its anwelcome appearance in Truro, among the cattle of a farmer of Comprigney. The fact of the outbreak having been re$30 r^{t}$ ed to the local sanitary authority, some surprise bas been expressed that no steps should have been taken to desare the place infected.

\section{ON STRAPPING THE CHEST IN PHTHISIS}

Br JOHN McCREA, M A., M.D., MERDICAL OFHICHR TO THM BHLPAST DISPBNSARY.

THE treatment of phthisis by restraining chest-movement deserves more attention than it has yet received. Partly for this reason, and partly to describe the appliance which I have latterly found most effective, $I$ wish again to direct inquiry to the subject.

In the large number of cases which have come before me in the practice of the Belfast Dispenaary, I have seen no remedy equal strapping the chest in efficiency and general applicability. At the same time the use of other remedies is not interfered with. The plasters used in strapping are quite able to bear the strain of walking and talking, so that gentle exercise and conversation are not forbidden; and, indeed, I have seen both rendered enjoyable where they had previously been irksome. I have not met with a case in any stage of the disease in which there was ground for attributing any bad result to the restraint of the chest. I say this because a paper on the subject threatened grave consequences if cases were not most thoughtfully selected after an exact measurement of the proportion of lung in volved. An extensive trial has convinced me that this dread is a dream and this refinement finical.

Since writing a paper which appeared in the November number of the Dublin Journal of Medical Science, I bave made an improvement in the apparatus, which diminishes the frequency of the renewal of the plasters and strengthens their grip. The following description contemplates their application to the upper part of the chest. I have principally used emplastrum roborans spread on swan's-down. The sheet, which is half a yard wide, is to be cut into transverse strips. Each strip is eighteen inches long; the breadth should be about three-quarters of an inch. The plasters should be only very slightly heated. The first strip runs up the back in the space between the spinal column and the posterior border of the scapula on the affected side, its starting-point being well below the level of the inferior angle of the scapula. It is to be applied gradually and deliberately, every portion being well rubbed in before the next portion is brought into contact with the skin. It is to be carried over the shoulder and down the front of the chest. In rounding the shoulder it is to be pulled tight and held so while it is being, bit by bit, brought into contact with the front of the chest, the chest just at this period being in the act of strong expiration. The next strip, which is horizontal, commences at the spine, crosses the posterior end of the first strip, passes under the axilla and on towards the sternum. It also is to be applied deliberately and with friction; as it is rounding the chest it is to be pulled tight, the patient at the same time making a forced expiration. Other strips are to be applied in a similar manner, vertically and borizontally time about, until it is judged that a proper grasp of the chest has been obtained. I avoid the scapula as much as possible. Some of the horizontal strips should cross the sternum, and some the spine. A large rectangular piece of plaster should now be applied, occupying the interscapular space and reaching down to the last dorsal spine. Another squarish piece is to cover the front and upper part of the chest between the clavicles and mamma. These, if smoothly applied, secure the ends of the strips from ruffing up, and give additional points d'appui. Finally, the whole is to be well rubbed in all over. The patient is to sit quiet for a few minutes before dressing. The plaster soils the fingers, which, however, may be easily cleaned by rubbing with coarse paper and washing with a few drops of ether. The length of strip required of course depends upon the size of the chest and the extent of the disease. I always endeavour to control more of the lung than the portion apparently diseased. I have found it generally suitable to cut the plaster as above described. If too long, that may be easily remedied with scissors as each strip is applied. If too short-if, for instance, a vertical plaster beginning on the back does not reach sufficiently far down the front of the chest, let the next vertical plaster commence its course in front and at a sufficiently low point, and then be made to cover the former. This, besides, increases the rigidity 
of the apparatus, and rigidity undoubtedly is one source of its power.

In a fortnight a reapplication will probably be required. This will give a good opportunity for a careful examination of the condition of the lung. While the plasters are still on the indications of the thermometer will be most valuable. If there be an exacerbation of the symptoms, particularly of the cough, dyspnoea, or pain, if the temperature rise, or if the plasters be obviously slack, apply new ones. In an advanced case of phthisis in a girl, the girl's mother told me that she herself could tell the proper time for renewal by observing the cough become distressing at night; and, indeed, it is common for patients to ask for a reapplication. This illustrates, besides, the confidence felt in the plasters by those who have had experience of their effects. In early phthisis it is necess'ry to warn the patients not to mistake the amelioration of their symptoms for recovery; they should always be directed to come back. Possibly when they consider themselves quite well the thermometer or the stethoscope will indicate differently. These are the cases in which, by reapplications, repeated reapplications if necessary, we may hope for the most brilliant results.

In the paper already referred to I have related a few cases, selected with the aim of illustrating the effects of this line of treatment in different stages of the disease. We obtain immediate and marked diminution of the cough, cessation of pain, relief of dyspnca, and reduction of temperature; and the patient usually expresses at once a feeling of great comfort. In short, I am so satisfied with the results of the numerous cases in which I have tried this method that I give it the first place among all the remedies for phthisis.

Papers on the subject. - Berkart on "Rest," \&c., THE LINCET, Oct. 18th, 1073; a letter from myself in the following number of the same journal; my paper in the Dublin Medical Journal, Novemher, 1873; Dobell on the "Importance and Dangers of Rest in Pulmonary Consumption," in the British Medical Journal, Nov. 22nd, 1873.

Belfast.

\section{SUBPERIOSTEAL EXCISION OF HUMERUS.}

\section{By J. A. DONOVAN, M.D.}

ON the morning of December 15th, 1872, while engaged in practice at Lewiston, Maine, U.S.A., I was hastily called to attend Daniel $\mathrm{M}$ - aged fifteen, who was said to be bleeding profusely from the nose. On reaching the boy's place of residence, it was ascertained that the hæmorrhage occurred first on the previous day, but ceased spontaneously. The second attack, being more severe, was treated by the application of solution of subsulphate of iron to the nasal mucous membranes and a little ice bag across the brows. The patient was fairly nourished and of medium development. The parents were healthy, as were a brother and sister, who comprised the family. A cousin died of phthisis, and other cousins manifested strumous tendencies. The patient's surroundings and home comforts were very satisfactory. He was now evidently very ill. The temperature, notwithstanding the loss of blood in considerable quantity, was elevated. The pulse was 130, and small in volume.

No appearance of local disease, to explain his constitutional disturbance, could be found, except a small patch of redness a little below and external to the coracoid process of the right scapula, corresponding with the neck of the humerus. There was a little swelling and heat at the inflamed part, and it hurt him to move the arm. The treatment was a suitable confinement of the arm to the body to ensure rest; and the extract of opium, made fluid with water, was freely painted over tbe inflamed part. He was given quinia with morphia sufficient to procure necessary sleep and freedom from pain.

Next day the patient appeared better, but the inflamed surface bad increased to double its extent of the day before; it was exceedingly tender to the touch, and was plainly an acute periostitis. His temperature appeared lower; pulse 118 ; appetite improved; and no return of the epistaxis. The parents, believing the case to be a mild attack of acute rheumatism, which was prevalent at that time in their locality, desired me to discontinue my attendance, and promised tu report if the patient did not progress farourably.
Four days later they did report that their boy was recovering satisfactorily.

On Dec. 25th, ten days after the first visit, I met with the patient's father, who informed me that the boy's arm was much swollen, but not painful ; and that he was feeble. He desired me to see the patient if deemed necessary. The arm was then found to be very tense and immensely swollen. It fluctuated freely from the top of the shoulder nearly to the elbow. The hand and forearm were very œdematous. The patient had emaciated rapidly; had had chills and perspirations; and by his appearance gave ample evidence of severe suffering, though he would not admit it. An incision through the soft parts, nearly corresponding with the patch of redness first observed, gave exit to a large quantity of ill-formed pus, containing numerous little clots of dark blood. The finger then came directly in contact with bare and finely-roughened bone. It could be passed around the neek of the humerus, upwards into the capsule, and downwards its length. The periosteum was detached extensively
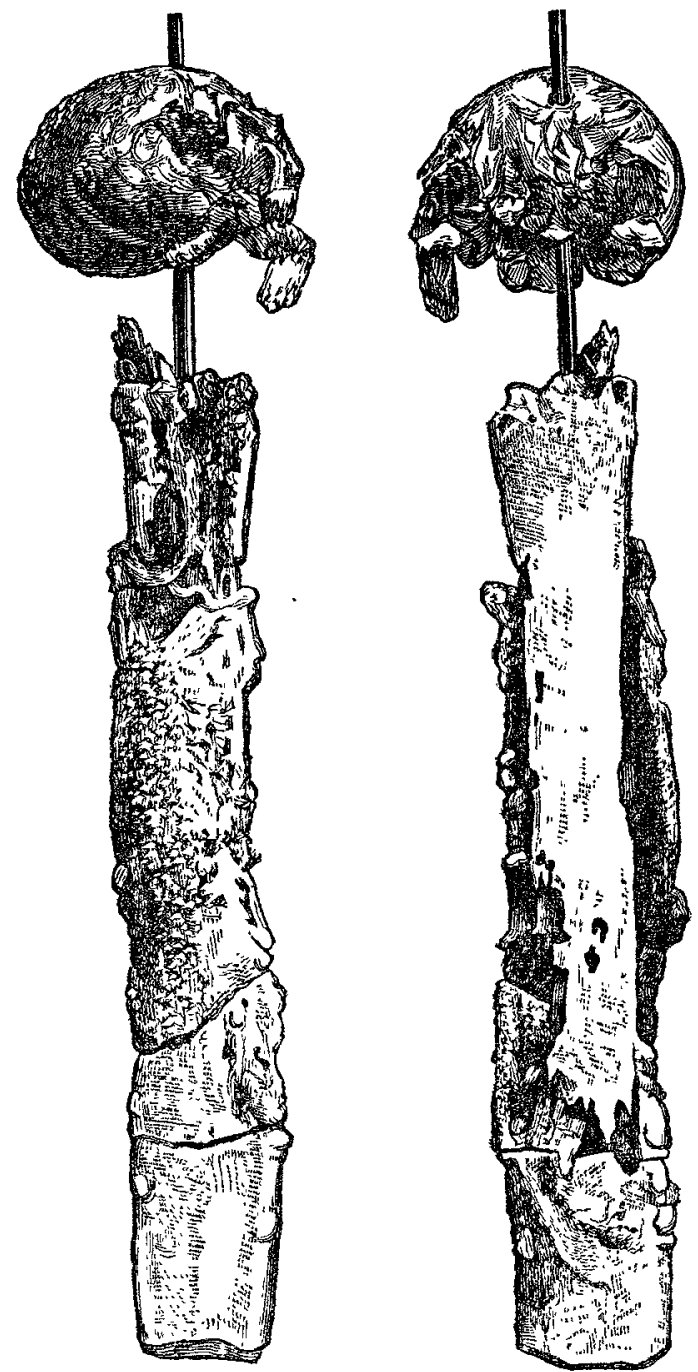

The above figures, half the original size, represent opposite surfaces of the bone, as well as different phases of the morbid proces The head and secterts morbid procss. The head and sere kept in relation by a wire passing through them. The interspace seen between the head and shaft shows as nearly ration.

from the outer and posterior surfaces of the bone. Three days later the patient was etherised, and another opening made between the condyles at the bottom of the carity. A long probe passed readily from the upper through the lower incision, and the pus drained completely away. He was then relieved of most of his pain.

The patient did well until Jan. 20th, 1873, when his arm began to be painful again, but not from confined pus. An intense erythematous redness soon diffused itself over the arm, accompanied by considerable swelling. The whole humerus seemed to be acting as a foreign body. Severe constitutional disturbance again appeared, having as principal features chills, perspirations, and diarrbca.

On Jan. 23rd it was decided to operate, and to perform the kind of operation that seemed most advisable after exposing the humerus. The incisions made for the escape of 